\title{
The Association between Microalbuminuria and Metabolic Syndrome in the General Population in Japan: The Takahata Study
}

\author{
Zhimei Hao ${ }^{1}$, Tsuneo Konta ${ }^{1}$, Satoshi Takasaki ${ }^{1}$, Hiroshi Abiko ${ }^{1}$, Mizue Ishikawa ${ }^{1}$, \\ Toshiyuki Takahashi ${ }^{1}$, Ami Ikeda ${ }^{1}$, Kazunobu Ichikawa ${ }^{1}$, Sumio Kawata ${ }^{2}$, \\ Takeo Kato ${ }^{3}$ and Isao Kubota ${ }^{1}$
}

\begin{abstract}
Objective The metabolic syndrome is associated with an increased risk of chronic kidney disease, cardiovascular disease and mortality. However, the association between microalbuminuria and the metabolic syndrome has not yet been reported in the general population in Japan. Therefore, we undertook a populationbased study to examine the association between microalbuminuria and the metabolic syndrome in Takahata, Japan.

Methods Subjects of this cross-sectional study were individuals aged from 40 to 87 years old. The metabolic syndrome was defined according to the criteria of the Adult Treatment Panel III. Microalbuminuria was defined as a urine albumin-creatinine ratio of 30 to $300 \mathrm{mg} / \mathrm{g}$.

Results A total of 2,321 subjects (mean age 64 years old) were entered into the final analysis. Among them, the prevalence of the metabolic syndrome and microalbuminuria was $16.5 \%$ and $13.7 \%$, respectively. There was a significantly positive correlation between the number of components of the metabolic syndrome and the corresponding prevalence of microalbuminuria $(\mathrm{p}<0.001)$. In the subjects with metabolic syndrome compared with those without metabolic syndrome, the age- and gender-adjusted odds ratio of microalbuminuria was 1.99 (95\% CI, 1.49-2.66). Multiple logistic regression analysis revealed that high glucose, high blood pressure and obesity were independently associated with microalbuminuria.

Conclusions Our study revealed a strong relationship between microalbuminuria and the metabolic syndrome in the general population in Japan. More comprehensive and intensive management of the metabolic syndrome at its early stage is important to prevent the progression of renal injury and cardiovascular complications.
\end{abstract}

Key words: chronic kidney disease, obesity, hypertension, diabetes, ATP III

(DOI: 10.2169/internalmedicine.46.6056)

\section{Introduction}

The metabolic syndrome, a multifactorial disorder, is characterized by abdominal obesity, hypertriglyceridemia, low high-density lipoprotein (HDL) cholesterol level, high blood pressure and high fasting glucose level. Recent works have shown that people with the metabolic syndrome based on the National Cholesterol Education Program (NCEP) Adult Treatment Panel III (ATP III) criteria are at an increased risk for diabetes, cardiovascular morbidity and mortality $(1,2)$.

Microalbuminuria is associated with an increased risk of end-stage renal disease, cardiovascular events and all-cause

\footnotetext{
${ }^{1}$ Department of Cardiology, Pulmonology, and Nephrology, Yamagata University School of Medicine, Yamagata, ${ }^{2}$ Department of Gastroenterology, Yamagata University School of Medicine, Yamagata and ${ }^{3}$ Department of Neurology, Hematology, Metabolism, Endocrinology and Diabetes, Yamagata University School of Medicine, Yamagata

Received for publication May 29, 2006; Accepted for publication August 20, 2006

Correspondence to Dr. Tsuneo Konta, kkonta@med.id.yamagata-u.ac.jp
} 
mortality. Several studies have disclosed that metabolic syndrome is independently associated with an increased risk for chronic kidney disease (CKD) and microalbuminuria among the general population of western countries $(3,4)$. In Japan, Tanaka et al showed that metabolic syndrome is a significant determinant of CKD in a hospital-based population (5). Recently we reported that a high prevalence of microalbuminuria was observed in the Japanese general population, especially among the elderly (6). However, the association between microalbuminuria and the metabolic syndrome has not been clarified in Japan before. Therefore, we undertook a population-based study to examine the association between microalbuminuria and the metabolic syndrome in Takahata, Japan.

\section{Methods}

\section{Study population}

This study is part of the ongoing Molecular Epidemiological Study utilizing the Regional Characteristics of $21 \mathrm{st}$ Century Centers of Excellence (COE) Program in Japan. The aim of the present study was to determine the association between microalbuminuria and the components of the metabolic syndrome in the general population in Japan. This study is a community-based and design-incorporated baseline survey that consisted of a self-administered questionnaire on lifestyle, blood pressure measurement, anthropometrical measurement, and collections of blood and urine specimens from participants in the morning.

The survey population in this study is the general population aged from 40 to 87 years in Takahata town located in the southern part of Yamagata prefecture, Japan. This region has a resident population of 15,222 adults over the age of 40 years (males: 7,109, females: 8,113). From June through November 2004, 1,055 males and 1,346 females (total, $2,401)$ took part in the program and agreed to join the study. This study was approved by the institutional ethical committee. All participants gave written informed consent.

Eighty subjects were excluded from the present analysis due to incomplete data. A total of 2,321 subjects entered into the final statistical analysis. There were 1,034 (44.5\%) males and 1,287 (55.5\%) females and the mean age was 64 years old.

\section{Measurement}

Subjects used a self-report questionnaire to document medical history, current medication, family history, clinical symptoms, smoking habits (current or non-smoker), and alcohol intake (current or non-drinker) . Systolic and diastolic blood pressures were determined by using a mercury manometer in a sitting position after at least 5 minutes rest. Measurement was performed twice, with the mean value used for statistical analysis. Body mass index (BMI) was calculated from weight and height measures as weight $(\mathrm{kg})$ divided by the square of height $\left(\mathrm{m}^{2}\right)$. Obesity was specified as $\mathrm{BMI} \geqq 25.0 \mathrm{~kg} / \mathrm{m}^{2}$ both in men and women (7). Urine albumin-creatinine ratio (UACR) was calculated from a single spot urine specimen collected in the morning. Urine albumin concentration was determined by immunoturbidimetry. Microalbuminuria and macroalbuminuria were defined as UACR 30 to $300 \mathrm{mg} / \mathrm{g}$ and $300 \mathrm{mg} / \mathrm{g}$, respectively by the National Kidney Foundation (8). Subjects were excluded when menstruation was present, because this renders the albumin measurement unreliable. Plasma glucose level was measured with a hexokinase enzymatic reference method. Serum HDL cholesterol, triglyceride and serum creatinine (SCr) were measured by enzymatic methods. Glomerular filtration rate (GFR) was estimated using the abbreviated Modification of Diet in Renal Disease (MDRD) study equation, including a correction factor of 0.742 for women (9). $\mathrm{SCr}$ was calibrated using the following formula:serum creatinine (Yaffe method) $=0.194+1.079 \times$ serum creatinine (enzymatic method) (5). To estimate GFR, we used calibrated SCr values. Chronic kidney disease was defined as micro- or macroalbuminuria among subjects with GFR of more than $60 \mathrm{ml} / \mathrm{min} / 1.73 \mathrm{~m}^{2}$ (CKD stage 1-2) and GFR of less than $60 \mathrm{ml} / \mathrm{min} / 1.73 \mathrm{~m}^{2}$ (CKD stage 3-5) (10). The metabolic syndrome was defined by modified National Cholesterol Education Program (NCEP) Adult Treatment Panel III (ATP III) guidelines criteria (11). Subjects were deemed to have metabolic syndrome if they had three or more of the following five criteria:1) obesity, BMI $\geqq 25 \mathrm{~kg} / \mathrm{m}^{2}, 2$ ) hypertriglyceridemia, triglycerides $\geqq 150 \mathrm{mg} / \mathrm{dl}$, 3) low HDL cholesterol $<40 \mathrm{mg} / \mathrm{dl}$ if male or $<50 \mathrm{mg} / \mathrm{dl}$ if female, 4) high blood pressure $\geqq 130 / 85 \mathrm{mmHg}$ or, 5) high fasting glucose $\geqq 110 \mathrm{mg} / \mathrm{dl}$. Participants who reported current use of antihypertensives or a treatment for diabetes were classified as having high blood pressure or high fasting glucose level, respectively. In this study we used BMI to classify subjects with obesity, because waist circumference measures were not available.

\section{Statistical analyses}

We used student's $t$-test to evaluate differences in means and chi-square tests to evaluate differences in proportions. The non-parametric Mann-Whitney $U$ test was utilized for the parameters of the data that were not normally distributed. To determine the odds ratio and $95 \%$ confidence intervals of microalbuminuria as a function of the metabolic syndrome, logistic regression analysis was used. The crude and age-, sex-adjusted odds ratios of microalbuminuria were determined by clustering the components of the metabolic syndrome. In these analyses, the odds ratios were calculated by comparing subjects with $1,2,3,4$, and 5 components of the metabolic syndrome with subjects without component. Next, the odds ratios were calculated by comparing subjects with the metabolic syndrome ( $\geqq 3$ components) and without the metabolic syndrome ( $<3$ components). Then, we used univariate analyses to examine the relationship between each component of the metabolic syndrome and UACR category. Finally, the odds ratios of microalbuminuria were calculated 
DOI: $10.2169 /$ internalmedicine.46.6056

Table 1. Characteristics of Subjects with and without Metabolic Syndrome

\begin{tabular}{|c|c|c|c|}
\hline Characteristic & $\begin{array}{l}\text { Subjects without MS } \\
\qquad(\mathrm{n}=1937)\end{array}$ & $\begin{array}{l}\text { Subjects with MS } \\
\qquad(\mathrm{n}=384)\end{array}$ & $\mathrm{p}$ value \\
\hline Age (yrs) & $63.6 \pm 10.3$ & $64.3 \pm 9.1$ & NS \\
\hline Men $(\%)$ & 44.2 & 46.4 & NS** \\
\hline Obesity (\%) & 21.5 & 79.4 & $<0.001 * *$ \\
\hline Body mass index $\left(\mathrm{kg} / \mathrm{m}^{2}\right)$ & $23.0 \pm 3.0$ & $26.5 \pm 2.6$ & $<0.001$ \\
\hline Triglyceride (mg/dl) & $86(27-696)$ & $166(41-743)$ & $<0.001 *$ \\
\hline HDLC (mg/dl) & $61.3 \pm 13.7$ & $46.1 \pm 11.2$ & $<0.001$ \\
\hline Systolic BP (mmHg) & $133.0 \pm 15.9$ & $141.1 \pm 12.7$ & $<0.001$ \\
\hline Diastolic BP (mmHg) & $78.1 \pm 9.8$ & $82.6 \pm 8.6$ & $<0.001$ \\
\hline Fasting plasma glucose (mg/dl) & $86.6 \pm 25.9$ & $99.2 \pm 35.6$ & $<0.001$ \\
\hline Current smoking (\%) & 31.4 & 31.5 & NS** \\
\hline Current drinking $(\%)$ & 40.4 & 39.3 & NS** \\
\hline Current medication for diabetes $(\%$ & 3.8 & 17.2 & $<0.001 * *$ \\
\hline Current medication for hypertensio & on $(\%)$ & 48.2 & $<0.001 * *$ \\
\hline Serum total cholesterol (mg/dl) & $199.6 \pm 30.6$ & $205.6 \pm 34.2$ & 0.001 \\
\hline Uric acid (mg/dl) & $4.96 \pm 1.31$ & $5.50 \pm 1.37$ & $<0.001$ \\
\hline Chronic Kidney Disease (\%) & 14.0 & 27.6 & $<0.001 * *$ \\
\hline Microalbuminuria (\%) & 12.2 & 20.8 & $<0.001 * *$ \\
\hline Macroalbuminuria (\%) & 1.1 & 4.7 & $<0.001 * *$ \\
\hline Urine creatinine (mg/dl) & $1.05(0.11-5.85)$ & $1.07(0.10-3.64)$ & NS* \\
\hline Urine albumin $(\mathrm{g} / \mathrm{dl})$ & $9.9(0.6-1654.5)$ & $13.9(0.8-6146)$ & $<0.001 *$ \\
\hline $\mathrm{UACR}(\mathrm{mg} / \mathrm{g})$ & $8.9(1.9-1151.8)$ & $12.7(2.1-5536.9)$ & $<0.001 *$ \\
\hline Serum creatinine $(\mathrm{mg} / \mathrm{dl})$ & $0.67 \pm 0.22$ & $0.70 \pm 0.18$ & 0.026 \\
\hline Estimated GFR $\left(\mathrm{ml} / \mathrm{min} / 1.73 \mathrm{~m}^{2}\right)$ & $77.8 \pm 12.8$ & $75.9 \pm 14.5$ & 0.007 \\
\hline
\end{tabular}

with each component of the metabolic syndrome as an exposure of interest separately using multivariate regression analysis. Data are expressed as mean \pm SD. All statistical analysis was performed using the software of Stat View version 5 (SAS Institute Inc, Cary, NC, USA) . A significant difference was defined as $\mathrm{p}<0.05$.

\section{Results}

\section{Characteristics of subjects}

Baseline characteristics of the 2,321 subjects that entered into the final analysis are shown in Table 1 . In total, $16.5 \%$ of the study participants had metabolic syndrome $(17.2 \%$ for men and $16.0 \%$ for women). The serum creatinine, uric acid, urine albumin, and urinary albumin-creatinine ratio were higher, while estimated GFR was lower among subjects with metabolic syndrome compared with those without metabolic syndrome. In subjects with metabolic syndrome, the prevalence of CKD, microalbuminuria or macroalbuminuria was significantly higher compared with those without metabolic syndrome.

Overall, $31.1 \%, 16.5 \%, 15.6 \%, 74.8 \%$ and $13.4 \%$ of the study sample had obesity, high triglyceride, low HDL cho- lesterol, high blood pressure and high fasting glucose, respectively. The prevalence of $0,1,2,3,4$ or 5 components of metabolic syndrome was $15.5 \%, 40.5 \%, 27.5 \%, 11.2 \%$, $4.4 \%$ and $0.9 \%$, respectively. Among the subjects with one component of the metabolic syndrome, the prevalence of obesity, high triglyceride, low HDL cholesterol, high blood pressure and high fasting glucose were $6.3 \%, 2.1 \%, 3.7 \%$, $84.5 \%$ and $3.4 \%$ respectively. In both men and women, high blood pressure was dominant among the components of the metabolic syndrome.

\section{Relationship between microalbuminuria and meta- bolic syndrome}

Next we investigated the relationship between microalbuminuria and the metabolic syndrome. Figure 1 shows the prevalence of microalbuminuria by the number of components of the metabolic syndrome. There was a significant positive correlation between the number of components present and the corresponding prevalence of microalbuminuria $(\mathrm{p}<0.001)$.

Table 2 shows the crude and age, gender-adjusted odds ratio of microalbuminuria associated with the number of components of the metabolic syndrome. In the adjusted model, subjects with $1,2,3,4$ and 5 components of the 
Table 2. Crude and Age- and Gender adjusted Odds Ratio of Microalbuminuria Associated with the Number of Components of Metabolic Syndrome

\begin{tabular}{|c|c|c|}
\hline \multirow{2}{*}{$\begin{array}{c}\text { No. of } \\
\text { Components }\end{array}$} & \multicolumn{2}{|c|}{ Odds ratio $(95 \% \mathrm{CI})$} \\
\hline & Crude & Age-, gender-adjusted \\
\hline $1 \S$ & $2.85(1.66-4.89)^{*}$ & $2.39(1.38-4.14)^{* *}$ \\
\hline $2 \S$ & $4.58(2.66-7.88)^{*}$ & $3.62(2.08-6.29)^{*}$ \\
\hline $3 \S$ & $5.24(2.91-9.46)^{*}$ & $4.46(2.45-8.12)^{*}$ \\
\hline $4 \S$ & $6.30(3.13-12.6)^{*}$ & $6.20(3.03-12.6)^{*}$ \\
\hline $5 \S$ & $15.9(5.88-43.4)^{*}$ & $13.3(4.65-38.1)^{*}$ \\
\hline MS\# & $1.98(1.49-2.62)^{*}$ & $1.99(1.49-2.66)^{*}$ \\
\hline
\end{tabular}

metabolic syndrome had increased odds of 2.39, 3.62, 4.46, 6.20 and 13.3 respectively, compared with those without component. Subjects with the metabolic syndrome had 1.99fold increased odds of microalbuminuria compared with their counterparts without the metabolic syndrome.

To examine the contribution of risk factors to the presence of microalbuminuria we used univariate analyses and multivariate logistic regression analyses. Univariate analyses revealed that the metabolic syndrome and each component of the metabolic syndrome were associated with increasing levels of UACR (Table 3) . Multivariate logistic regression analyses revealed the odds ratios for microalbuminuria given specific components of the metabolic syndrome (Table 4). On multivariate analyses, obesity, high glucose and high blood pressure were independently associated with microalbuminuria.

\section{Discussion}

In this study, the prevalence of metabolic syndrome in general population was $16.5 \%$. This value was higher than those of previous reports $(6.0-12.0 \%)$ among the Japanese young population (mean age less than 50 years old) $(5,12)$. It is already known that the prevalence of metabolic syndrome increases with aging. However, our result is still much lower than those of other studies among young population in Europe and United States, e.g., 23.6\% in Greece (13) and $23.1 \%$ in NHANES III (14). Although this difference might have been partly derived of the differences in definitions used for the metabolic syndrome, the ethnic properties including dietary pattern and genetic predisposition might have a great effect on the metabolic syndrome. It is reported that the cutoffs for BMI or abdominal girth to identify high-risk subjects may be influenced by ethnicity due to the differential sensitivity to the deleterious effects of obesity among ethnic groups (15). Notably, the morbidity and mortality of the Asian population is increasing in people with a lower BMI as compared with those of Caucasians (7).

In the young population, obesity or dyslipidemia is a

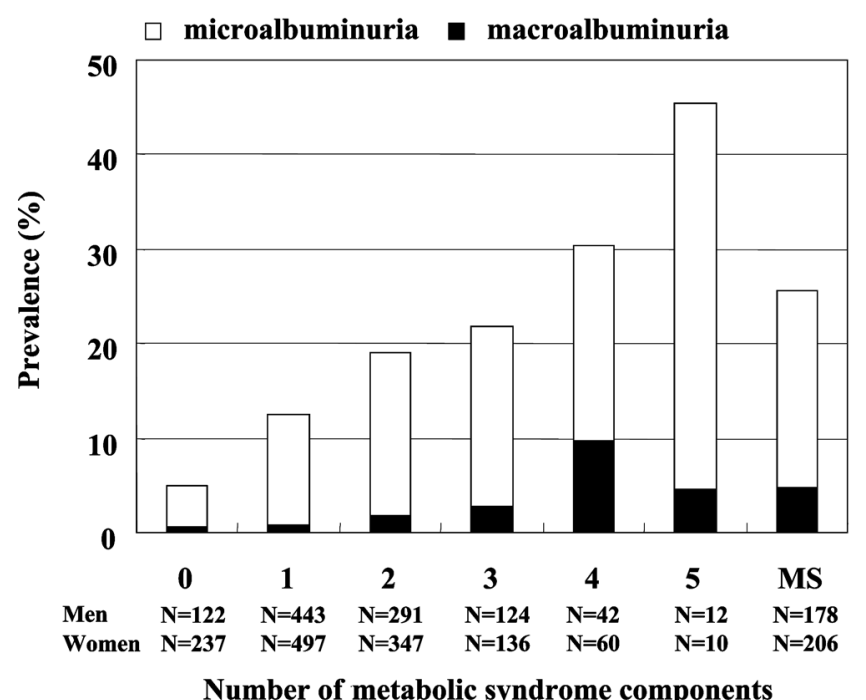

Figure 1. Prevalence of microalbuminuria according to the number of metabolic syndrome components. MS, metabolic syndrom.

leading component of metabolic syndrome and the prevalence of high blood pressure is less than $50 \%$ in other reports including a Japanese study $(4,5,13)$. By contrast, in our relatively elderly population, high blood pressure was the most predominant component of metabolic syndrome. The same trends were found among men or women. These results indicate that in the middle-aged and elderly population in Japan, high blood pressure is likely the most frequent component of metabolic syndrome which leads a substantial number of subjects to the pre-stage of metabolic syndrome.

The prevalence of micro- and macroalbuminuria increased with the number of components of metabolic syndrome (Fig. 1). Importantly, the prevalence of microalbuminuria was much higher than that of macroalbuminuria. This indicates that a large number of subjects with microalbuminuria are easily overlooked in routine health checkups that do not include a microalbuminuria examination. The increase in microalbuminuria was significant even in the subjects with 1 or 2 components of metabolic syndrome (Table 2). Therefore, it is suggested that intervention for metabolic syndrome should be started at the earliest opportunity to prevent the progression of renal injury.

In the present study, in the middle-aged and elderly general subjects, all of the components of metabolic syndrome were associated with microalbuminuria. In particular, high plasma glucose, high blood pressure and obesity were the major risk factors for microalbuminuria, as previously reported $(3,16)$. The subclinical level of increase in blood pressure and plasma glucose is typical in the relatively older population. Thus, it should be stressed that these factors might be strongly associated with microalbuminuria in this population.

There are several limitations in our study. First, we used BMI $\geqq 25$ to classify individuals with obesity, because waist 
Table 3. Prevalence of the Components of Metabolic Syndrome in UACR category

\begin{tabular}{lcccc}
\hline \multicolumn{4}{c}{ UACR category } \\
Components of & $\begin{array}{c}\text { Normoalbuminuria } \\
(n=1965)\end{array}$ & $\begin{array}{c}\text { Microalbuminuria } \\
(n=317)\end{array}$ & $\begin{array}{c}\text { Macroalbuminuria } \\
(n=39)\end{array}$ & p value \\
\hline MS & 29.2 & 39.7 & 56.4 & $<0.001$ \\
Higesity (\%) & 15.2 & 22.4 & 30.8 & $<0.001$ \\
Low HDLC (\%) & 15.1 & 17.0 & 30.8 & 0.021 \\
High BP (\%) & 72.5 & 87.4 & 89.7 & $<0.001$ \\
High glucose (\%) & 10.8 & 26.2 & 38.5 & $<0.001$ \\
\hline MS (\%) & 14.6 & 25.2 & 46.2 & $<0.001$ \\
\hline Chi-square test; UACR, urine albumin-creatinine ratio; TG, triglyceride; HDLC, high-density lipoprotein cholesterol; BP, blood pressure; \\
normoalbuminuria, microalbuminuria and macroalbuminuria were defined as UACR $<30 \mathrm{mg} / \mathrm{g}, 30$ to $300 \mathrm{mg} / \mathrm{g}$ and $>300 \mathrm{mg} / \mathrm{g} ;$ \\
MS, metabolic syndrome
\end{tabular}

Table 4. Odds Ratio of Microalbuminuria by Components of Metabolic Syndrome

\begin{tabular}{lccc}
\hline & Odds Ratio & $(95 \% \mathrm{CI})$ & $\mathrm{p}$ value \\
\hline Obesity & 1.31 & $(1.01-1.70)$ & 0.037 \\
High TG & 1.34 & $(0.97-1.85)$ & $\mathrm{NS}$ \\
Low HDLC & 0.91 & $(0.64-1.30)$ & $\mathrm{NS}$ \\
High BP & 2.37 & $(1.66-3.36)$ & $<0.001$ \\
High glucose & 2.64 & $(1.97-3.55)$ & $<0.001$ \\
\hline TG, triglyceride; HDLC, high-density lipoprotein cholesterol; BP, blood pressure; CI, confidence interval; \\
NS, not significant
\end{tabular}

circumference measures were not available. This might have caused an underestimation of the effect of obesity on microalbuminuria (17). However, recent studies have used BMI successfully to classify those with metabolic syndrome (18, $19)$, lending support to the validity of our measure. In particular, the report of Shiwaku et al stated that the definition of BMI $\geqq 25$ as obesity is suitable for the determination of the metabolic syndrome among Japanese (20). Second, because this is a cross-sectional study, the measurement of microalbuminuria was carried out only once, which did not completely conform to the requirement of multiple urine collections for microalbuminuria (21). Therefore, it is necessary to determine the incidence of microalbuminuria by prospective observation in those free from metabolic syndrome. Third, in Takahata town, the majority of persons working at a company undergo a medical examination at their workplace. They were not covered by our study. Furthermore, only persons who are interested in health care came to this screening and persons that are under current medication or uninterested in health care did not come. Therefore, there is a possibility that the participant bias might affect these results.

In conclusion, the present study revealed a strong relationship between microalbuminuria and metabolic syndrome in the general population in Japan. More comprehensive and intensive management of the metabolic syndrome at its early stage should be started to prevent the progression of renal injury and cardiovascular complications.

Sources of support:A grant-in-aid from the 21st century center of excellence (COE) program of the Japan Society for the Promotion of Science and a grant-in-aid for Scientific Research (No. 14770546) from the Ministry of Education, Science, Sports and Culture, Japan.

\section{References}

1. Laaksonen DE, Lakka HM, Niskanen LK, Kaplan GA, Salonen JT, Lakka TA. Metabolic syndrome and development of diabetes mellitus: application and validation of recently suggested definitions of the metabolic syndrome in a prospective cohort study. Am J Epidemiol 156: 1070-1077, 2002.

2. Isomaa $\mathrm{B}$, Almgren $\mathrm{P}$, Tuomi $\mathrm{T}$, et al. Cardiovascular morbidity and mortality associated with the metabolic syndrome. Diabetes Care 24: 683-689, 2001.

3. Chen J, Muntner P, Hamm LL, et al. The metabolic syndrome and chronic kidney disease in U.S. adults. Ann Intern Med 140: 167174, 2004.

4. Kurella M, Lo JC, Chertow GM. Metabolic syndrome and the risk for chronic kidney disease among nondiabetic adults. J Am Soc Nephrol 16: 2134-2140, 2005.

5. Tanaka H, Shiohira Y, Uezu Y, Higa A, Iseki K. Metabolic syndrome and chronic kidney disease in Okinawa, Japan. Kidney Int 69: 369-374, 2006.
6. Konta T, Hao Z, Abiko H, et al. Prevalence and risk factor analysis of microalbuminuria in Japanese general population: The Takahata Study. Kidney Int 70: 751-775, 2006.

7. WHO/IASO/IOTF. The Asia-Pacific Perspective: Redefining Obesity and its Treatment. Health Communications. Australia, Pty Ltd, 2000: $15-21$.

8. Keane WF, Eknoyan G. Proteinuia, albuminuria, risk, assessment, detection, elimination (PARADE) : a position paper of the National Kidney Foundation. Am J Kidney Dis 33: 1004-1010, 1999.

9. Levey AS, Bosch JP, Lewis JB, Greene T, Rogers N, Roth D. A more accurate method to estimate glomerular filtration rate from serum creatinine:A new prediction equation. Modification of Diet in Renal Disease Study Group. Ann Intern Med 130: 461-470, 1999.

10. National Kidney. Foundation. K/DOQI clinical practice guidelines of chronic kidney disease: Evaluation, classification, and stratification. Am J Kidney Dis 39 (suppl 2): S46-S75, 2002. 
11. Expert Panel, on Detection. Evaluation, and Treatment of High Blood Cholesterol in Adults. Executive Summary of The Third Report of The National Cholesterol Education Program (NCEP) Expert Panel on Detection, Evaluation, and Treatment of High Blood Cholesterol in Adults (Adult Treatment Panel III). JAMA 285: 2486-2497, 2001.

12. Enkhmaa B, Shiwaku K, Anuurad E, et al. Prevalence of the metabolic syndrome using the Third Report of the National Cholesterol Educational Program Expert Panel on Detection, Evaluation, and Treatment of High Blood Cholesterol in Adults (ATP III) and the modified ATP III definitions for Japanese and Mongolians. Clin Chim Acta 352: 105-113, 2005.

13. Athyros VG, Bouloukos VI, Pehlivanidis AN, et al. The prevalence of the metabolic syndrome in Greece: The MetS-Greece Multicentre Study. Diabetes Obes Metab 7: 397-405, 2005.

14. Ford ES, Giles WH, Mokdad AH. Increasing prevalence of the metabolic syndrome among U.S. Adults. Diabetes Care 27: 24442449, 2004.

15. Fernandez JR, Allison DB. Understanding racial differences in obesity and metabolic syndrome traits. Nutr Rev 61: 316-319, 2003.
16. Palaniappan L, Carnethon M, Fortmann SP. Association between microalbuminuria and the metabolic syndrome:NHANES III. Am J Hypertens 16: 952-958, 2003.

17. Horie N, Komiya H, Mori Y, Tajima N. New body mass index criteria of central obesity for male Japanese. Tohoku J Exp Med 208: 83-86, 2006.

18. Malik S, Wong ND, Franklin SS, et al. Impact of the metabolic syndrome on mortality from coronary heart disease, cardiovascular disease, and all causes in United States adults. Circulation 110: 1245-1250, 2004.

19. Wong ND, Sciammarella MG, Polk D, et al. The metabolic syndrome, diabetes, and subclinical atherosclerosis assessed by coronary calcium. J Am Coll Cardiol 41: 1547-1553, 2003.

20. Shiwaku K, Nogi A, Kitajima K, et al. Prevalence of the metabolic syndrome using the modified ATP III definitions for workers in Japan, Korea and Mongolia. J Occup Health 47: 126-135, 2005.

21. Levey AS, Eckardt KU, Tsukamoto Y, et al. Definition and classification of chronic kidney disease: a position statement from Kidney Disease:Improving Global Outcomes (KDIGO). Kidney Int 67: 2089-2100, 2005.

(C) 2007 The Japanese Society of Internal Medicine http://www.naika.or.jp/imindex.html 